Revue d'histoire de l'Amérique française

DAS REVUE D.HISTOIRE DE L'AMÉRIQUE FRANÇAISE

\title{
Les Inuits du Labrador méridional face à l'exploitation canadienne et française des pêcheries (1700-1760)
}

\section{François Trudel}

Volume 31, numéro 4, mars 1978

URI : https://id.erudit.org/iderudit/303648ar

DOI : https://doi.org/10.7202/303648ar

Aller au sommaire du numéro

Éditeur(s)

Institut d'histoire de l'Amérique française

ISSN

0035-2357 (imprimé)

1492-1383 (numérique)

Découvrir la revue

Citer cet article

Trudel, F. (1978). Les Inuits du Labrador méridional face à l'exploitation

canadienne et française des pêcheries (1700-1760). Revue d'histoire de

l'Amérique française, 31(4), 481-499. https://doi.org/10.7202/303648ar d'utilisation que vous pouvez consulter en ligne. 


\title{
LES INUIT DU LABRADOR MÉRIDIONAL FACE À L'EXPLOITATION CANADIENNE ET FRANÇAISE DES PÊCHERIES (1700-1760)*
}

François Trudel

Département d'anthropologie

Université Laval

\begin{abstract}
«Autrefois les Eskimaux montaient jusqu'à Mingan, mais les établissements qui se sont fait le long de la côte les ont obligé de s'éloigner». - Jean-Louis Fornel, 1743
\end{abstract}

\section{Le problème}

«Heureux sont les peuples sans histoire». C'est ainsi qu'un ethnologue canadien réputé, Diamond Jenness, faisait débuter il y a quelque dix années un ouvrage sur l'administration coloniale chez les Inuit du Labrador. Jenness voulait par ces quelques mots caractériser une période d'administration par les missionnaires moraves (de 1771 à 1915) au cours de laquelle les Inuit du Labrador étaient demeurés en vase clos, «ignorés du reste du monde et l'ignorant». Mais il cherchait aussi à établir un contraste entre cette période et la précédente, qui fut marquée par des événements notoires.

* Une première version de cet article, réalisé grâce à l'aide financière du Conseil des Arts du Canada, a été présentée au Congrès annuel de l'Institut d'histoire de l'Amérique française, à l'Université de Sherbrooke, le 8 octobre 1976. Le terme vernaculaire «Inuk»: un homme, «Inuit»: les hommes, est ici préféré aux termes «Esquimaux », "Eskimaux », quoique ces derniers puissent apparaître dans les citations. La côte du Labrador méridional s'étend des îles et îlets de Mingan (CôteNord du St-Laurent) jusqu'à la baye des Esquimaux ou Hamilton Inlet (Labrador actuel). Source de la citation: J.-L. Fornel, «Relation de la découverte qu'a faite le Sieur Fornel en 1743 de la Baye des Eskimaux » in Rapport de l'Archiviste de la Province de Québec [à l'avenir RAPQ] (1920-21): 73. M. Michel Guénet a dressé la carte illustrant cet article. 
Selon Jenness, en effet, dès le $16^{\mathrm{e}}$ siècle, des pêcheurs européens commencent à visiter régulièrement le Labrador méridional et se risquent même à y hiverner à partir du début du $17^{\mathrm{e}}$ siècle. Ils sont suivis par des commerçants qui exploitent les ressources poissonnières de cette côte et qui tentent d'y établir des relations avec les Inuit tout au cours du $17^{\mathrm{e}}$ siècle et la première partie du $18^{\mathrm{e}}$ siècle. Des échanges sporadiques ont alors lieu, permettant aux Inuit d'obtenir certains objets manufacturés. Toutefois, très tôt au $17^{\mathrm{e}}$ siècle, une méfiance réciproque se développe entre les Inuit et les Européens qui sont alliés aux Montagnais, et se transforme vite en hostilités ouvertes. Les Inuit, incapables d'offrir une résistance efficace contre leurs adversaires mieux armés, sont obligés de se retirer progressivement vers le nord à partir du début du $18^{\mathrm{e}}$ siècle $^{1}$. Bref, aux $17^{\mathrm{e}}$ et $18^{\mathrm{e}}$ siècles, les Inuit du Labrador méridional n'apparaissent pas comme un peuple «sans histoire». Au contraire, il y a là pour eux une histoire réelle et longue: celle de l'abandon d'une côte où ils entrent en contact avec les Européens et en conflit avec les Montagnais.

Les ouvrages historiques et ethnologiques qui se rapportent à la côte du Labrador expliquent en partie ce recul des Inuit. Packard fait intervenir des facteurs climatiques et surtout des facteurs ethnologiques, soit leur extermination par les Indiens ${ }^{2}$. Gosling met aussi en évidence l'hostilité qui existait entre les Montagnais et les Inuit du Labrador. Il explique le retrait progressif de ces derniers par la série de défaites que leur firent subir les Montagnais, appuyés par les Français ${ }^{3}$. Cette interprétation a été reprise par Hawkes, Speck et Tanner ${ }^{4}$. Ce dernier souligne qu'aux $17^{\mathrm{e}}$ et $18^{\mathrm{e}}$ siècles, les Indiens et les Blancs pillèrent et décimèrent les groupes d'Inuit qu'ils rencontraient sur la côte. Les Inuit auraient résisté à ces attaques pendant un certain temps, mais durent finalement retraiter vers le détroit de Belle-Isle et l'Atlantique lorsque les Français fournirent des

1 D. Jenness, Eskimo Administration, III, Labrador, Arctic Institute of North America, Technical Paper no 16 (Montreal, 1965): 7-8.

2 A. S. Packard, The Labrador Coast (New York, 1891), 246, 259.

3 W. G. Gosling, Labrador: its discovery, exploration and development (London, A. Rivers, 1910), 133, 166.

4 F. W. Hawkes, The Labrador Eskimo, Memoir 91, Canada, Department of Mines (Ottawa, 1910): 16-17; F. G. Speck, "Inland Eskimo Bands of Labrador», in Essays in Anthropology in Honor of A. L. Kroeber (University of California Press, 1936), 321, 325: V. Tanner, «Outlines of the Geography, Life and Customs of Newfoundland and Labrador», in Acta Geographica 8 (1) (Helsinki, 1944): 479-481. 
armes à feu à leurs alliés. Ceci rejoint les explications de Jenness qui insiste davantage toutefois sur la rancune des Inuit envers les Français ${ }^{5}$.

$\mathrm{Si}$ on écarte l'hypothèse de changements climatiques importants, que Packard n'a pas réussi à démontrer, toutes les explications mettent de l'avant l'hostilité traditionnelle entre Inuit et Montagnais, hostilité attisée par l'appui des Français à ces derniers. Nous croyons que ces explications restent insuffisantes. En imputant aux Amérindiens la majeure partie de la responsabilité dans l'expulsion des Inuit, ces interprétations sous-estiment l'importance de la présence des Européens au Labrador à cette époque.

Ainsi, le recul des Inuit au Labrador méridional apparaît plutôt comme la conséquence directe de l'exploitation des pêcheries par les Canadiens et les Français. Peu à peu, ceux-ci s'approprient le territoire du Labrador méridional et finissent par en expulser les Inuit. L'analyse de cette expulsion au cours de la période 1700-1760 doit donc commencer par l'étude approfondie des pêcheries canadiennes et françaises et de leur développement.

\section{L'exploitation canadienne et française au Labrador méridional}

Dès le $16^{\mathrm{e}}$ siècle et même avant Jacques Cartier, des pêcheurs français vinrent en bon nombre pratiquer la pêche sur les côtes de Terre-Neuve et du Labrador méridional ${ }^{6}$. Ils purent s'y livrer à une exploitation intensive au premier endroit, mais furent bientôt obligés d'abandonner le deuxième, en raison des dépradations faites aux installations de pêche par les Inuit au cours du $17^{\mathrm{e}}$ siècle $^{7}$. L'état de guerre qui sévit entre la France et l'Angleterre à la fin de ce siècle se prolonge jusqu'au traité d'Utrecht alors que la France cède la baie et le détroit d'Hudson, Terre-Neuve et toute l'Acadie péninsulaire ${ }^{8}$. Dès lors, le Labrador offre un intérêt grandissant pour l'exploitation des pêcheries, un fait sur lequel Packard, Tanner ou même Innis n'ont pas suffisamment insisté.

5 D. Jenness, op. cit.: 7-11.

6 H. A. Innis, The Cod Fisheries: The History of an International Economy (Yale University Press, New Haven, 1940), 11-26.

7 Ch. de la Morandière, Histoire de la pêche française de la morue dans l'Amérique septentrionale, Tome II (Éditions G. P. Maisonneuve et Larose, Paris, 1962): 706.

8 M. Trudel, Initiation à la Nouvelle-France, Histoire et Institutions (Holt, Rinehart and Winston, Montréal, 1968), 81. 
TABLEAU I

Liste des concessions et concessionnaire sur la côte méridionale du Labrador entre 1661 et 1760

\begin{tabular}{|c|c|c|c|}
\hline Lieux de concession & $\begin{array}{l}\text { Date de la } \\
\text { concession ou de } \\
\text { la prolongation }\end{array}$ & Concessionnaire & $\begin{array}{l}\text { Identification } \\
\text { sommaire }\end{array}$ \\
\hline \multirow{3}{*}{$\begin{array}{l}\text { Mingan } \\
\text { Anticosti }\end{array}$} & $1661(1)$ & Bissot & Juge prévôt \\
\hline & 1676 & Radisson & $\begin{array}{l}\text { Explorateur, } \\
\text { aspirant de la } \\
\text { marine }\end{array}$ \\
\hline & $1679-80(2)$ & $\begin{array}{l}\text { Des Groseillers } \\
\text { Lalande }\end{array}$ & Explorateur \\
\hline \multirow{2}{*}{ Mingan et Anticosti } & & Jolliet & \\
\hline & 1703 & Jolliet (fils) & $\begin{array}{l}\text { Marchand et } \\
\text { traiteur }\end{array}$ \\
\hline Itamamiou & 1733 & Belcour & -, C.S. (3) \\
\hline Petit M̄ecatina & 1740 & De St-Vincent & Officier miiitaire \\
\hline Grand Mecatina & $1738-1739-1749(4)$ & Pommereau & Écrivain \\
\hline Kecarpoui & 1748 & $\begin{array}{l}\text { Bréard } \\
\text { Estèbe }\end{array}$ & $\begin{array}{l}\text { Contrôleur de la } \\
\text { marine, C.S. } \\
\text { Garde-magasin du } \\
\text { roi }\end{array}$ \\
\hline \multirow[t]{5}{*}{ Riv. des Esquimaux } & $\begin{array}{l}1702(5) \\
1706\end{array}$ & $\begin{array}{l}\text { Courtemanche } \\
\text { Amador de } \\
\text { Godefroy }\end{array}$ & $\begin{array}{l}\text { Officier militaire } \\
\text { Écuyer }\end{array}$ \\
\hline & 1720 & Lavaltrie & - \\
\hline & 1750 & Cheron & Capitaine de navire \\
\hline & 1753 & De Cery & Capitaine de port \\
\hline & 1761 & De Cery (fils) & \\
\hline \multirow[t]{2}{*}{ Shecatica } & 1738 & $\begin{array}{l}\text { Foucault } \\
\text { Boucault }\end{array}$ & $\begin{array}{l}\text { Marchand, C.S. } \\
\text { Marchand, } \\
\text { procureur du roi }\end{array}$ \\
\hline & 1750 & Lafontaine & - \\
\hline \multirow[t]{3}{*}{ Baye de Phélypeaux } & $\begin{array}{l}1711 \\
1714\end{array}$ & $\begin{array}{l}\text { Courtemanche } \\
\text { Brouage (fils) }\end{array}$ & $\begin{array}{l}\text { Officier militaire } \\
\text { Négociant, officier } \\
\text { militaire }\end{array}$ \\
\hline & 1722 & Brouage & $\begin{array}{l}\text { Négociant, officier } \\
\text { militaire }\end{array}$ \\
\hline & 1744 & La Porte & $\begin{array}{l}\text { Commissaire de la } \\
\text { marine }\end{array}$ \\
\hline Petit Hâvre & 1750 & Marsal & Négociant \\
\hline Blanc Sablon & 1689 & La Chesnaye (6) & Marchand \\
\hline \multirow[t]{2}{*}{ Anse Ste-Claire } & 1750 & Taché & Négociant \\
\hline & 1751 & Fortier & Marchand \\
\hline La $F$ & 1748 & Vincent & Capitaine de navire \\
\hline Anse-à-Loup & 1748 & Deschenaux & Écrivain \\
\hline
\end{tabular}




\begin{tabular}{|c|c|c|c|}
\hline Rivière des Français & 1716 & Constantin & Pêcheur et traiteur \\
\hline & 1751 & Bréard & $\begin{array}{l}\text { Contrôleur de } \\
\text { marine, C.S. }\end{array}$ \\
\hline St-Modet & 1735 & Foucault & Marchand, C.S. \\
\hline & & Boucault & $\begin{array}{l}\text { Marchand, } \\
\text { procureur du roi }\end{array}$ \\
\hline & 1740 & Constantin & Pêcheur et traiteur \\
\hline Baye des Châteaux & 1735 & Bazil & Négociant \\
\hline & 1736 & Bazil & Négociant \\
\hline & & Fornel & Marchand \\
\hline & $\begin{array}{l}1749 \\
1735\end{array}$ & Gaultier & Médecin du roi, C.S. \\
\hline Cap Charles & $\begin{array}{l}1735 \\
1744\end{array}$ & $\begin{array}{l}\text { Riverin } \\
\text { Marsal }\end{array}$ & Marchand, C.S. \\
\hline & 1749 & De Bonne & Militaire \\
\hline & 1754 & Marsal & Négociant \\
\hline Belle Isle & 1689 & Riverin (7) & Marchand, C.S. \\
\hline $\begin{array}{l}\text { Passage des Loups- } \\
\text { marins }\end{array}$ & $\begin{array}{l}1713(8) \\
1721\end{array}$ & $\begin{array}{l}\text { Constantin } \\
\text { De Leigne }\end{array}$ & $\begin{array}{l}\text { Pêcheur et traiteur } \\
\text { Officier de la } \\
\text { Prévôté }\end{array}$ \\
\hline Baye des Esquimaux & 1706 & $\begin{array}{l}\text { Amador de } \\
\text { Godefroy }\end{array}$ & Écuyer \\
\hline & 1739 & Daine & Greffier en chef \\
\hline & & Foucault & Négociant, C.S. \\
\hline & 1749 & Fornel (veuve) & \\
\hline Terre-Neuve & 1705 & Hazeur & Marchand, C.S. \\
\hline
\end{tabular}

NOTES: (1) De l'Isle-aux-Oeufs à la baye des Espagnols (ou de Phélypeaux).

(2) Jusqu'à la baye de Phélypeaux.

(3) C.S. - Conseil supérieur.

(4) En 1749, concédé à LeGardeur de Croisille et de Montesson, époux de la veuve de Pommereau, ainsi qu'à De Beaujeu et Estèbe.

(5) De la rivière Kégaska à celle de Kessessakiou.

(6) Ainsi que d'autres marchands - Pachot, Poisset, Martin de Lino, Lallemant, Pattu, Gobin.

(7) En plus des marchands Chanion, Catignon, Bouthier.

(8) Du détroit de Belle-Isle à la baye des Esquimaux.

Le Roi souligne en effet en 1713 que l'établissement de l'île Royale «ne serait pas suffisant pour occuper tous les Navires du Royaume qui font le Commerce de la pesche et qu'il est nécessaire de profiter des avantages qu'on peut tirer de la Côte de l'abrador $»^{9}$. En conséquence, il ordonne d'explorer cette côte en détail. Cela donne lieu à un mémoire qui énumère les diverses ressources du Labrador, suggère la fondation de 3 ou 4 établissements côtiers et

9 «Mémoire de Roi à Vaudreuil et Bégon», 25 juin 1713, in RAPQ (1947-48): 
met en évidence la présence d'Inuit sur cette côte ainsi que les services qu'ils pourraient rendre aux Français s'ils étaient pacifiés ${ }^{10}$. Un supplément à ce même mémoire, écrit en 1717, conclut que «le Labrador est un grand pays dont l'État peut retirer de grands avantages, qu'il est très important de s'y bien établir et de le mettre en valeur $»^{11}$.

Il n'en tenait qu'à lui. La côte méridionale du Labrador faisait partie du domaine royal et, sur la recommandation des gouverneurs et intendants de la colonie, le Roi pouvait concéder certaines parties de ce territoire. D'ailleurs, de vastes portions de la côte avaient déjà été concédées «en seigneuries», donc à perpétuité (voir le Tableau 1$)^{12}$.

À partir de 1713 , le nombre de concessions augmente, mais il s'agit seulement de fermages ou locations pour un temps limité, variant de 10 à 20 ans, et l'étendue des territoires excède rarement 4 à 5 lieues de front. Des prolongations et des augmentations de ces mêmes concessions sont aussi faites tout au long de la période 17131760.

Les concessions sont accordées à des marchands ou à des officiers civils ou militaires intéressés au commerce et qui font généralement partie de l'élite de la ville de Québec. Elles impliquent pour leurs titulaires des obligations et des droits qu'il est important de connaitre pour comprendre la nature de cette occupation. Aux exigences habituelles concernant la conservation des bois, la découverte de ressources minérales, les chemins et passages, s'ajoutent pour le concessionnaire des obligations plus spécifiques. Il doit payer au Domaine de sa Majesté une redevance annuelle de deux à cinq castors d'hiver ou une somme variant de quatre à dix livres. Il est aussi parfois tenu de laisser les pêcheurs utiliser les grèves

10 Anonyme, «Mémoire concernant le Labrador», 1715, in Archives des Colonies [à l'avenir AC], C11A, vol. 109: 50ss. ibid. : 78.

11 Anonyme, «Supplément pour mon mémoire touchant le Labrador», 1717,

12 Sources des données sur le régime des concessions: P.-G. Roy, éd., Inventaire de Pièces sur la côte de Labrador conservées aux Archives de la Province de Québec (Québec, 1940, 1942), 2 volumes; Great Britain, Privy Council, In the Matter of the Boundary between the Dominion of Canada and the Colony of Newfoundland in the Labrador Peninsula, vol. VII, (1927): 3716-3725; Dictionnaire biographique du Canada, tomes I, II, III (Les Presses de l'Université Laval, Québec, 1966, 1969, 1974). 
qu'il n'occupe pas. Enfin, si le titre ne lui accorde pas un droit exclusif sur la pêche à la morue, il lui donne par contre le monopole de la pêche au phoque dans l'étendue de la concession ainsi que le droit exclusif à la traite des fourrures avec les Amérindiens et les Inuit.

L'octroi de ces titres de concession par le Roi entraîne une occupation effective des côtes du Labrador et une activité économique importante fondée sur la pêche et la traite.

Plusieurs documents signalent l'abondance des phoques dans cette région et insistent sur leur valeur commerciale. Dès 1705 , on note le succès du poste de Courtemanche «par la grosse quantité d'huile qu'on peut y faire tant de loups-marins que de vaches marines ${ }^{13}$. Les préférences des Canadiens vont aux phoques plutôt qu'aux baleines, parce que l'huile de phoque vaut plus cher et est assurée d'un débit annuel possible de 2000 barriques, sans compter que les peaux sont vendues aux tanneurs ${ }^{14}$.

Pour mettre en valeur cette ressource, des postes de pêche sont établis tout au long de la côte, depuis Mingan jusqu'au cap Charles (voir la carte). D'abord exploités uniquement l'été, lorsque les phoques migrent vers l'Atlantique par le détroit de Belle-Isle, ces postes en viennent vite à rester ouverts jusqu'au début de l'hiver, au moment où les phoques longent le littoral pour aller séjourner près de l'île Anticosti. La pêche qu'on pratique dans ces postes est une pêche sédentaire, différente de la chasse. Elle se fait avec des filets tendus à flot entre les îles et le littoral et exige des préparatifs considérables commençant dès le mois d'avril, pour la pêche estivale, et dès le mois de septembre, pour la pêche hivernale. La pêche ellemême a lieu du 20 juin au 6 juillet et du 15 décembre au 6 janvier, après quoi la graisse de phoque est fondue et mise en barriques, les peaux sont grattées et salées pour être expédiées à Québec pendant la saison de navigation ${ }^{15}$.

13 "Beauharnois et Raudot au Ministre», 19 octobre 1705, in RAPQ (193839): 87. Le terme utilisé par les Français au début du $18^{\mathrm{e}}$ siècle est «loup-marin». Nous préférons ici le terme scientifique, qui comprend aussi bien les phoques du Groenland (Pagophilus groenlandicus) que les phoques à capuchon (Cystophora cristata). Voir A. W. Mansfield, Seals of Arctic and Eastern Canada, Fisheries Research Board of Canada (Ottawa, 1967).

14 «Projet de l'Instruction... au Gouverneur général et à l'Intendant du Canada», 16 juin 1716, in AC, C11A, vol. 122 (1): 159; Anonyme, "Mémoire sur la pêche et la chasse au Canada", 1723, in AC, C11A, vol. 122: 277.

15 P. Beaucage, "Technologie de la pêche au loup-marin sur la Côte-Nord du St-Laurent ", in L'Homme 8 (3) (1968): 96-125. Voir cet article pour une description détaillée de toute la technologie de la pêche au phoque. 


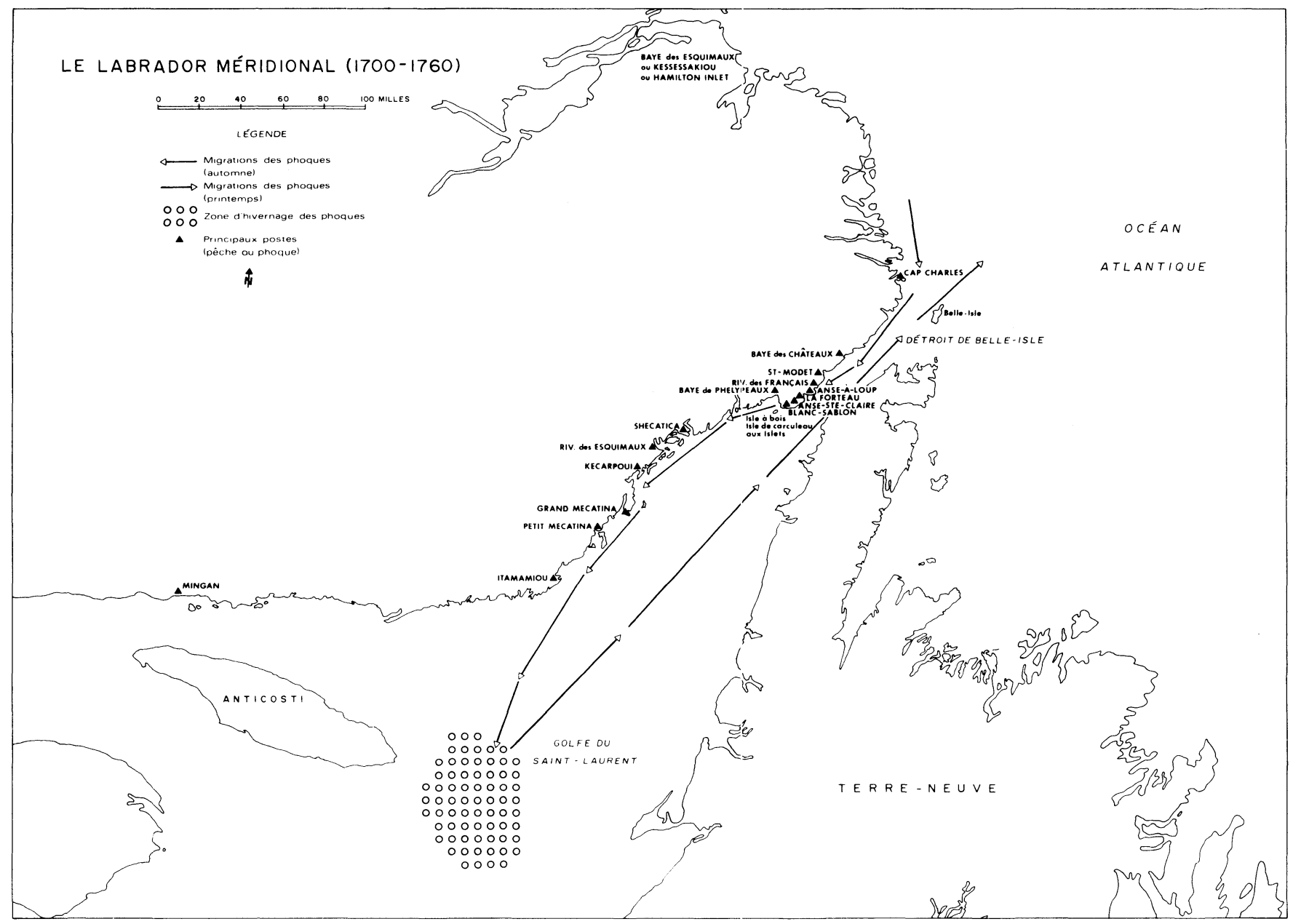


TABLEAU II

État des postes de pêche sédentaire au phoque sur la côte méridionale du Labrador (vers 1760)

\begin{tabular}{|c|c|c|c|c|}
\hline Poste & Engagés & $\begin{array}{l}\text { Période de } \\
\text { la pêche }\end{array}$ & Barriques d'huile & Peaux de phoque \\
\hline $\begin{array}{l}\text { Nontagamiou } \\
\text { Grand Mecatina } \\
\text { Riv. des Esquimaux } \\
\text { Shécatica } \\
\text { Baye de Phélypeaux } \\
\text { La Forteau } \\
\text { Lance à Loup } \\
\text { St-Modet } \\
\text { Baye Rouge } \\
\text { Baye des Chateaux } \\
\text { Cap Charles }\end{array}$ & $\begin{array}{l}10 \\
25 \\
12 \\
10 \\
50 \\
12 \\
25 \\
\\
15 \\
15\end{array}$ & $\begin{array}{l}15 \text { déc-6 janv. } \\
15 \text { déc-6 janv. } \\
15 \text { déc- } 6 \text { janv. } \\
15 \text { déc- } 6 \text { janv. } \\
20 \text { juin-10 juillet } \\
20 \text { juin-6 juillet } \\
15 \text { déc-6 janv. } \\
15 \text { déc-6 janv. } \\
15 \text { déc- } 6 \text { janv. } \\
15 \text { déc- } 6 \text { janv. }\end{array}$ & $\begin{array}{c}80-100 \\
400-600 \\
50-80 \\
80-120 \\
400-500 \\
200-250 \\
\\
200-300 \\
\\
\\
150-300 \\
150-300\end{array}$ & 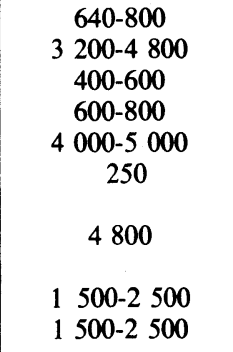 \\
\hline TOTAUX & 174 & & $\begin{array}{l}\operatorname{minimum}: 1710 \\
\operatorname{maximum}: 2550\end{array}$ & $\begin{array}{l}\text { minimum: } 16890 \\
\text { maximum: } 22050\end{array}$ \\
\hline
\end{tabular}

Source: «État des postes établis sur la Coste du Nord concédés par brevet de la cour et qui sont exploités actuellement et leurs productions", in RAPQ 19331934: 218 .

Note: La période de la pêche indiquée ci-haut correspond aux moments de l'année durant lesquels les phoques sont pêchés avec des filets. La préparation de la pêche sédentaire au phoque s'étend sur une période beaucoup plus longue.

Les concessionnaires résident parfois en permanence à leurs postes pour faire effectuer ce travail ${ }^{16}$, mais ils ont le plus souvent recours à des maîtres de poste qui dirigent des engagés originaires de la région de Québec, travaillant à contrat et recevant pour gages une part de la production ${ }^{17}$. Certains maitres de poste utilisent aussi de la main-d'œuvre montagnaise ${ }^{18}$. Étant donné l'ensemble des activités reliées à la pêche au phoque elle-même et à sa préparation, les maîtres de poste et leurs engagés occupent la côte de façon permanente, été comme hiver, même si les effectifs peuvent être plus réduits durant cette dernière saison. En dehors des saisons de pêche, les engagés sont occupés à la chasse et à l'équarrissage, ainsi qu'à la traite avec les autochtones ${ }^{19}$.

16 Comme par exemple Courtemanche à la rivière des Esquimaux et Brouage à la baye de Phélypeaux.

17 P.-G. Roy, op. cit., vol. II.

18 Anonyme, "Mémoire concernant le Labrador», op. cit.: 54.

19 J.-N. Fauteux, Essai sur l'Industrie au Canada sous le régime français (Québec, 1927), 502. 
TABLEAU III

États annuels des navires qui font la pêche sédentaire à la morue sur la côte du Labrador

\begin{tabular}{|c|c|c|c|c|c|c|c|c|c|c|c|c|c|c|c|c|}
\hline \multirow[t]{2}{*}{ Années } & \multicolumn{4}{|c|}{1718} & \multicolumn{4}{|c|}{1726} & \multicolumn{4}{|c|}{1729} & \multicolumn{4}{|c|}{1731} \\
\hline & 1 & 2 & 3 & & 1 & 2 & 3 & & 1 & 2 & 3 & & 1 & 2 & 3 & 4 \\
\hline ISLE-À-BOIS & 12 & 890 & 1965 & & 3 & 230 & 10100 & & 9 & 725 & 17700 & & 9 & 793 & 20100 & 193 \\
\hline BLANC-SABLON & 6 & 271 & 772 & & - & - & - & & 2 & 149 & 3500 & & 1 & 105 & 2600 & 24 \\
\hline LA FORTEAU & 8 & 515 & 1002 & & 5 & 338 & 10900 & & 3 & 205 & 6000 & & 5 & 283 & 6600 & 67 \\
\hline ANSE-À-LOUP & 3 & 210 & 400 & & 4 & 270 & 10000 & & 3 & 171 & 4800 & & 3 & 225 & 3700 & 51 \\
\hline SAINT-MODET & 5 & 273 & 575 & & 3 & 170 & 60010 & & 1 & 25 & 1000 & & - & - & - & - \\
\hline AUX ISLETS & 3 & 46 & - & & - & - & - & & - & - & - & & - & - & - & - \\
\hline ISLE-AUX-MARMETTES & - & - & - & & - & - & - & & - & - & 一 & & - & - & - & - \\
\hline ISLE DE CARCULEAU & - & - & - & & - & - & - & & - & - & & & - & - & - & - \\
\hline TOTAUX ANNUELS & 37 & 2205 & 4714 & & 15 & 1008 & 3700 & & 18 & 1275 & 33000 & & 18 & 1406 & 33000 & 335 \\
\hline \multirow[t]{2}{*}{ Années } & \multicolumn{4}{|c|}{1732} & \multicolumn{4}{|c|}{1733} & \multicolumn{4}{|c|}{1736} & \multicolumn{4}{|c|}{1736} \\
\hline & 1 & 2 & 3 & 4 & 1 & 2 & 3 & 4 & 1 & 2 & 3 & 4 & 1 & 2 & 3 & 4 \\
\hline ISLE-À-BOIS & 7 & 582 & 4490 & 237 & 6 & 587 & 12000 & 376 & 7 & 675 & 28000 & 440 & 7 & 532 & 26500 & 289 \\
\hline BLANC-SABLON & - & - & - & - & 2 & 144 & 5300 & 108 & 3 & 245 & 7700 & 135 & 1 & 150 & 6000 & 72 \\
\hline LA FORTEAU & 4 & 390 & 1190 & 132 & 3 & 292 & 9900 & 192 & 3 & 285 & 10100 & 170 & 4 & 239 & 12500 & 130 \\
\hline ANSE-À-LOUP & 3 & 235 & 560 & 80 & 3 & 170 & $57(10$ & 133 & 2 & 150 & 4800 & 85 & 2 & 140 & 7000 & 83 \\
\hline SAINT-MODET & - & - & - & - & - & - & - & - & - & - & - & & - & - & - & - \\
\hline AUX ISLETS & - & - & - & - & - & - & - & - & - & - & - & - & - & - & - & - \\
\hline ISLE-AUX-MARMETTES & 1 & 100 & 330 & 32 & 1 & 76 & 3500 & 70 & - & - & - & - & 1 & 80 & 4000 & 47 \\
\hline ISLE DE CARCULEAU & 一 & 一 & - & 一 & 一 & 一 & - & - & 1 & 110 & 4000 & 70 & 一 & - & - & 一 \\
\hline TOTAUX ANNUELS & 15 & 1307 & 7877 & 481 & 15 & 209 & 36400 & 879 & 16 & 1465 & 54600 & 900 & 15 & 1141 & 56000 & 621 \\
\hline \multirow[t]{2}{*}{ Années } & \multicolumn{4}{|c|}{1738} & \multicolumn{4}{|c|}{1739} & \multicolumn{4}{|c|}{1742} & \multicolumn{4}{|c|}{1743} \\
\hline & 1 & 2 & 3 & 4 & 1 & 2 & 3 & 4 & 1 & 2 & 3 & 4 & 1 & 2 & 3 & 4 \\
\hline ISLE-À-BOIS & 5 & 395 & 19000 & 265 & 8 & 523 & 21800 & 269 & 7 & 583 & 26300 & 223 & 4 & 264 & 15200 & 161 \\
\hline BLANC-SABLON & - & - & - & - & 1 & 84 & 3300 & 42 & - & - & - & - & - & - & - & - \\
\hline LA FORTEAU & 4 & 220 & 9000 & 135 & 3 & 201 & 5100 & 103 & 5 & 290 & 12900 & 117 & 4 & 345 & 17800 & 179 \\
\hline ANSE-À-LOUP & 3 & 225 & 9000 & 150 & 4 & 243 & 11100 & 137 & 4 & 288 & 13500 & 124 & 5 & 295 & 15600 & 159 \\
\hline SAINT-MODET & - & 一 & - & - & - & - & - & - & - & - & - & - & - & - & - & - \\
\hline AUX ISLETS & - & - & - & - & - & - & - & - & - & - & - & - & - & - & - & - \\
\hline ISLE-AUX-MARMETTES & - & - & - & - & - & 一 & - & - & - & - & - & - & - & - & - & - \\
\hline ISLE DE CARCULEAU & 3 & 245 & 11500 & 175 & 1 & 122 & 4500 & 60 & 1 & 70 & 3000 & 27 & 1 & 96 & 5000 & 50 \\
\hline TOTAUX A & 15 & 085 & 48500 & 725 & 17 & 173 & 76000 & 521 & 17 & 231 & 55700 & 491 & 4 & 00 & 600 & \\
\hline
\end{tabular}


ISLE-À-BOIS

BLANC-SABLON

23

34

LA FORTEAI

ANSE-À-LOUP

SAINT-MODET

AUX ISLETS

ISLE-AUX-MARMETTES

ISLE DE CARCULEAU

TOTAUX ANNUELS

\begin{tabular}{|rrrr|}
\hline 84 & 6779 & $\begin{array}{r}196700 \\
(6455)\end{array}$ & 2453 \\
16 & 1148 & 28400 & 381
\end{tabular}

$16 \quad 1148 \quad 28400$

(772)

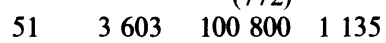

$39 \quad 2622 \quad(2192)$

$\begin{array}{rlll}2622 & 85200 & 1002\end{array}$

$(960)$

$3 \quad 46 \quad(575)$

\begin{tabular}{l|ccccc}
\hline TOTAUX ANNUELS & 212 & 15565 & 453600 & 9408 \\
$(323)$ & $(16991)$ & $(11284)$ &
\end{tabular}

LÉGENDE: 1- nombre de navires; 2- n. d'hommes; 3 - n. de quintaux de morue ; 4- n. de barriques d'huile. Les données sur la quantité de morue pêchée en 1718 et 1732 sont en milliers de morue et sont indiquées entre parenthèses dans la colonne «totaux» no. 3 .

SOURCE: "États des navires qui ont fait la pêche de morue sèche à la côte de Labrador", in RAPQ 1922-1923: 359ss.

NOTES: En plus des données figurant dans ce tableau, nous savons d'après les mêmes «états" que 4 navires fréquentèrent la côte du Labrador en $1712 ; 5$ en $1713 ; 11$ en $1714 ; 27$ en 1719; 19 en 1720, avec 1426 hommes; 17 en 1721 et 28 en 1728. Ces données additionnelles figurent avec un astérisque dans les colonnes 1 et 2 des totaux. Isle-à-Bois, Aux Islets, Isle de Carculeau et probablement aussi l'Isle-aux-Marmettes sont situées près du détroit de Belle-Isle. 
Si les données sur la production de ces pêcheries sédentaires au phoque sont éparses, elles révèlent une présence continue et une activité assez intense: 1000 barriques en 1735,600 en 1736, 810 en 1737, 2218 en 1744, 1884 en 1745, 1503 en $1746^{20}$. De plus, les postes de Grand Mécatina, baye de Phélypeaux, Saint-Modet/baye Rouge sont plus importants que les autres, tant par le nombre d'engagés que par le volume de la production d'huile et de peaux de phoques (voir le Tableau II) ${ }^{21}$. Ils occupent 100 hommes sur un total de 174 et fournissent les deux tiers de la production de l'ensemble des postes de pêche au phoque sur la côte du Labrador méridional ${ }^{22}$. Il est significatif de noter que deux de ces postes sont situés dans le détroit de Belle-Isle.

La traite avec les Amérindiens et les Inuit est une autre activité des Canadiens, même si des morutiers français s'y adonnent aussi occasionnellement. Les concessionnaires et leurs employés entretiennent surtout des relations régulières de traite avec les Montagnais de la Côte Nord du Saint-Laurent, avec qui ils échangent des peaux de castor. La traite avec les Inuit, quant à elle, n'atteint pas un niveau comparable d'activité. Les échanges entre Canadiens et Inuit restent sporadiques, fortuits et ne s'établissent jamais sur une base régulière. Ils constituent une activité économique marginale en regard de la pêche sédentaire au phoque et à la morue.

La pêche à la morue est surtout pratiquée par les morutiers français. Après le traité d'Utrecht, ils réapparaissent en nombre sur la côte du Labrador. La rareté des grèves appropriées pour sécher la morue et la concurrence entre les navires entraînent une diminution du nombre de bâtiments qui se stabilise à environ une quinzaine d'unités par année jusqu'en 1743. Au total, on dénombre un minimum de 323 visites de navires sur cette côte durant la première moitié du $18^{\mathrm{e}}$ siècle (cf. Tableau III) ${ }^{23}$.

20 J. Hamelin, Économie et Société en Nouvelle-France, Cahiers de l'Institut d'Histoire no 3 (Les Presses de l'Université Laval, Québec, 1960), 33.

21 Anonyme, "État des postes établis sur la coste du Nord concédés par brevet de la cour, et qui sont exploités actuellement, et leur production », non daté, in RAPQ (1933-34): 218.

22 Leur production est de 1000 barriques d'huile de phoque sur un minimum total de 1710 ; de 1400 barriques sur un maximum total de 2550 ; de 12000 peaux sur un minimum total de 16890 ; de 14600 peaux sur un maximum total de 22050 .

23 Brouage, «États des navires qui ont fait la pêche à la côte de Labrador», 1713 et après, in RAPQ (1922-23): 359ss. 
Ces navires se distribuent sur la côte du Labrador en un nombre limité de hâvres insulaires et littoraux, situés pour la plupart dans le détroit de Belle-Isle. L'Isle-à-Bois, Blanc-Sablon, La Forteau et l'Anse-à-Loup reçoivent des visites annuelles régulières de navires tout au cours de la première moitié du $18^{\mathrm{e}}$ siècle. Quatre emplacements voisins retiennent aussi l'attention des pêcheurs, quoique moins régulièrement, soit Saint-Modet, aux Islets, Isle-aux-Marmette et Isle de Carculeau. Au total, c'est le hâvre de l'Isle-à-bois qui reçoit le plus grand nombre de navires (84), suivi du hâvre de la Forteau (51), de l'Anse-à-Loup (39) et de celui de Blanc-Sablon (16). Les autres hâvres ne reçoivent au total que 22 navires.

Même si les données sont incomplètes il n'y a pas de doute que la pêche à la morue occupe davantage d'hommes que la pêche aux phoques. Il n'y a jamais moins de 1000 hommes annuellement et il se trouve une année, 1718, où on en compte plus de 2000 .

La production de cette pêche sédentaire se divise en morue sèche et en huile de foie de morue. Pour les années où les données sont disponibles la production de morue sèche, calculée en quintaux et parfois en milliers $(1718,1732)$ apparaît assez régulière, quel que soit le nombre de navires et d'hommes. Elle atteint un total de 453600 quintaux (en plus de 11284 milliers de morue) soit une moyenne annuelle de 41236 quintaux. La production d'huile de foie de morue, une activité complémentaire des morutiers français, est quant à elle de 9048 barriques, soit une moyenne annuelle de 104 barriques $^{24}$. Dans l'une et l'autre production, c'est l'Isle-à-Bois qui apparaît la plus productive avec presque le double de la production de morue séchée et au-delà du double de la production d'huile du hâvre le plus productif suivant, La Forteau.

Dans la première moitié du $18^{\mathrm{e}}$ siècle, les ressources de la côte du Labrador attirent l'attention des coloniaux et des métropolitains. Des marchands et des officiers de la Nouvelle-France, hommes d'affaires avant tout, cherchent à tirer profit de la pêche au phoque. Ils obtiennent des concessions du Roi et créent une série de postes de pêche sédentaire. Pour assurer la production, ils font appel à une main-d'œuvre coloniale qui s'établit sur les territoires reçus en concession. À cette présence des Canadiens s'en ajoute une autre, encore

24 Pour une description des méthodes de préparation de l'huile de foie de morue, consulter N. Denys, Histoire naturelle de l'Amérique septentrionale, traduite et annotée par W. F. Ganong (The Champlain Society, Toronto, 1908), 289. 
plus importante: celle de morutiers français qui pratiquent la pêche sédentaire à la morue. Ainsi de 1713 à 1760 , plus d'un millier d'hommes occupent de façon régulière et continue le littoral du Labrador méridional durant la saison estivale. De plus, les pêcheries les plus productives sont concentrées près du détroit de Belle-Isle, le véritable centre de la pêche sur cette côte et aussi le lieu privilégié par les Inuit pour l'obtention d'objets manufacturés. Il faut maintenant voir l'impact de l'exploitation canadienne et française des pêcheries sur ce groupe autochtone.

\section{Exploitation canadienne et française des pêcheries et expulsion des Inuit}

L'arrivée des Européens dans la région du détroit de Belle-Isle bouleversa la répartition traditionnelle des populations amérindiennes du pourtour du golfe du St-Laurent. Les possibilités de traite avec les pêcheurs européens attirèrent plusieurs d'entre elles sur la côte méridionale du Labrador. Au 16e siècle, Cartier rencontra des Amérindiens originaires du sud (peut-être des Micmacs) qui faisaient la chasse au phoque près de Blanc-Sablon. À Natashquan, il vit aussi d'autres Amérindiens (peut-être des Montagnais) qui revenaient eux-mêmes du secteur occidental du détroit de Belle-Isle où ils avaient vu des navires chargés de poissons appareiller vers l'Europe $^{25}$. A la fin du $16^{\mathrm{e}}$ siècle et au début du $17^{\mathrm{e}}$ siècle, les Inuit envahirent à leur tour le détroit de Belle-Isle ${ }^{26}$. Ils y vinrent vite aux prises avec les Amérindiens et avec les pêcheurs européens et forcèrent au moins ceux-ci à abandonner la côte méridionale du Labrador. L'établissement de postes de pêche sédentaire sur cette côte au $18^{\mathrm{e}}$ siècle allait donc poser de façon aiguë toute la question de son occupation et de la nature des rapports entre Inuit et pêcheurs, dont dépendait le sort du développement des pêcheries canadiennes et françaises.

Selon Gosling et Tanner, les Canadiens et les Français rencontrèrent au début du $18^{\mathrm{e}}$ siècle un nombre considérable d'Inuit jusqu'à Anticosti ${ }^{27}$. Ceux-ci n'y auraient été, selon nous, que des visiteurs

25 Ch.-A. Julien, éd., «Premier voyage de Cartier en 1534 », dans Les Français en Amérique pendant la première moitié du XVIe siècle (Les Presses universitaires de France, Paris, 1946), 87-88, 111.

26 W. G. Gosling, op. cit., 165-166.

27 Ibid., 166; V. Tanner, op. cit.: 481. Dans le cadre limité de cet article, nous ne prétendons pas solutionner la question complexe des limites de l'occupation des Inuit sur la Côte-Nord du St-Laurent. 
saisonniers. Courtemanche rapporte en effet que c'est sur la côte atlantique du Labrador méridional que «les Esquimaux font leur résidence à cause de la commodité de la vie qui s'y trouve jusqu'au Kessessakiou et même par delà, tout cet espace est bordé d'îles habitées par les Esquimaux; toutes les îles sont remplies de loupsmarins, de vaches marines et de toute sorte de gibier ${ }^{28}$. Fornel confirme cette observation dans sa «Relation de la découverte de la Baye des Eskimaux» en 1743. Il y a fait la distinction entre ce qu'il appelle la «coste de Labrador», qui s'étend de Mingan jusqu'au cap Charles, et la «coste des Esquimaux», i.e. la côte atlantique du Labrador. Il ajoute que les «Sauvages Eskimaux sont répandus depuis le détroit de Bel-Isle le long de la mer jusqu'au détroit d'Hudson», qu'ils ont un village à environ douze lieues de la baye StLouis (ou des Eskimaux) et que c'est à partir de ce point qu'ils se répandent le long de la coste». Dans une baie située au nord du cap Charles, nommée "baye des Meniques», Fornel entre de plus en contact avec plusieurs Inuit et commente leur présence à cet endroit de la façon suivante: "le grand nombre d'Eskimaux que nous avons trouvé dans la baye des Meniques donnent lieu de croire qu'ils y étaient comme en rendez-vous, d'où ils se disposaient d'aller faire leur pillage le long de la coste de Labrador; ils attendaient que les pêcheurs fussent partis ${ }^{29}$.

Ces observations des contemporains s'ajoutent à ce que nous savons sur les migrations annuelles des phoques et nous amènent à brosser un tableau de la localisation et du rythme de vie des Inuit à cette époque. Les Inuit occupent surtout la côte atlantique du Labrador méridional. L'été et plus souvent l'automne, une partie d'entre eux s'aventure dans le détroit de Belle-Isle et le long de la Côte-Nord du Saint-Laurent pour y obtenir avant tout des objets manufacturés auprès des pêcheurs canadiens et français. Ils peuvent y rester durant l'hiver, faisant la chasse au phoque et même la chasse au caribou le long des côtes $^{30}$. Au printemps, ils retournent sur la côte atlantique du Labrador méridional.

${ }^{28}$ Courtemanche, «Description de la Coste des Esquimaux», non daté, in AC, C11A, vol. 109: 13.

29 J.-L. Fornel, «Relation...de la Baye des Eskimaux», op. cit. : 73-74.

30 Notons que c'est à la fin de l'automne et au début de l'hiver que les phoques du Groenland et les phoques à capuchon - une des principales ressources alimentaires et vestimentaires des Inuit - longent la côte du Labrador méridional et vont hiverner près de l'île Anticosti. Ces phoques repartent vers le nord à la fin du printemps, en se dirigeant cette fois-ci directement vers le détroit de Belle-Isle (voir 
Il est impossible de connaître le nombre d'Inuit qui se déploient ainsi. En octobre 1716, Courtemanche évalue leur nombre à 400 tant à la Forteau qu'à Blanc-Sablon ${ }^{31}$. Avec le développement progressif des pêcheries canadiennes et françaises dans le détroit de BelleIsle, ce nombre diminue peu à peu, ainsi qu'en font foi quelques témoignages de Brouage et d'autres concessionnaires ${ }^{32}$.

Ce sont les relations entre les pêcheurs et les Inuit qui entrấnent cette diminution. Dès le début, les relations entre les deux groupes sont tendues. La bonne volonté de quelques entrepreneurs ne suffit pas à renverser le climat d'hostilité qui se propage rapidement. Bientôt, l'intérêt économique des pêcheurs canadiens et français prévaut.

En théorie, les Français souhaitent développer des relations amicales avec les Inuit. Il faut, dit-on, «empêcher les Montagnais et autres Amérindiens de faire la guerre aux Inuit et défendre aux Français de tirer sur eux; les attirer, les faire s'établir proche des postes et être juste dans les échanges qu'on peut avoir avec eux; leur donner à manger; engager des missionnaires pour les apprivoiser». Ils estiment toutefois qu'une fois pacifiés, les Inuit pourraient rendre de grands services aux Français, soit en les aidant directement dans leurs pêcheries, soit en leur échangeant divers produits. En pratique, l'attitude des pêcheurs varie d'un extrême à l'autre: «les uns pour les rendre traitables veulent essayer de les attirer et de lier commerce, d'autres pour les éloigner de leurs postes feront sur eux des actes d'hostilité $»^{33}$.

Par ailleurs, le comportement des Inuit ne favorise pas l'établissement de bonnes relations. À défaut de pouvoir procéder à des échanges, ils s'approprient tout simplement les objets manufacturés et les produits qu'ils convoitent. De plus, ils se livrent à un pillage constant de l'équipement et des agrès de pêche, volent les chaloupes et prennent ou brûlent le bois utilisés pour les séchoirs à morue. Ils attaquent aussi parfois les postes de pêche, tuant plusieurs employés. De 1715 à 1728 , de tels incidents se produisent presque cha-

la carte). La présence du caribou sur les rives du détroit de Belle-Isle est signalée par C. W. Townsend, ed., Captain Cartwright and his Labrador Journal (Dana Estes and Co., Boston, 1911), 72.

31 «M. Lair à Mme Courtemanche», 16 octobre 1716, in AC, C11A, vol. 109: 86.

32 Brouage, "Mémoires au Conseil de Marine sur ce qui s'est passé à la Côte de Labrador depuis le départ des derniers vaisseaux ", in RAPQ (1922-23): 359ss.

33 Anonyme, «Mémoire concernant le Labrador», op. cit.: 59ss. 
que année; par la suite, ils diminuent et ne sont plus commis que dans les hâvres les plus éloignés du détroit de Belle-Isle ${ }^{34}$.

En fait, quel que soit l'angle sous lequel on les regarde, les activités des pêcheurs canadiens et français et celles des Inuit sont incompatibles. Les concessionnaires canadiens et leurs employés exploitent le phoque, qui est à la base même du mode d'exploitation des Inuit, à l'aide d'une main-d'œuvre montagnaise. Ils traitent principalement avec les Montagnais, qui sont hostiles aux Inuit. Privés de la possibilité d'obtenir des objets manufacturés par des voies pacifiques, les Inuit ne craignent pas de recourir au vol et au pillage des postes et emplacements de pêche des Canadiens et des morutiers français, s'exposant ainsi à leurs représailles.

Celles-ci sont immédiates et drastiques et ne tardent pas à avoir des effets sur la présence des Inuit sur la Côte-Nord du St-Laurent et dans le détroit de Belle-Isle. Nommés par le Roi «commandants de la Côte du Labrador» et investis de la mission de protéger les pêcheries de cette côte, Courtemanche et Brouage construisent des établissements fortifiés respectivement à la rivière des Esquimaux en 1702, et à la baye de Phélypeaux en 1714. Ils affrontent eux-mêmes les Inuit à quelques occasions et font des prisonniers qu'ils gardent à leurs postes ${ }^{35}$. On retrouve d'autres prisonniers au Canada: une femme et un jeune garçon en 1729, une jeune Inuk à Beauport en 1732, une douzaine d'esclaves Inuit dans la seule ville de Québec ${ }^{36}$. Jusqu'en 1740, les morutiers français sont encore plus impitoyables. Ils tuent pour protéger leur équipement de pêche: "Quand des équipages voyaient des sauvages s'approcher, ils tiraient dessus de peur d'être massacrés eux-mêmes sans s'occuper de savoir s'il s'agissait d'Esquimaux ou d'autres indigènes ${ }^{37}$. Un léger changement se fait sentir à partir de 1740: pour contrer les larcins des Inuit, les morutiers français ne réagissent plus qu'en leur donnant la bastonnade et des coups de poing ${ }^{38}$.

34 Sources de ces données: «M. Lair à Mme Courtemanche», op. cit.: 83; Brouage, "Mémoires au Conseil de Marine...», op. cit.: 359ss; Anonyme, "Note sur les Esquimaux », 1757, in RAPQ (1923-24): 8.

35 Brouage, "Mémoire au Conseil de Marine...», 9 sept. 1718, in RAPQ (192223): 360 .

36 Brouage, «Mémoire au Conseil de Marine...», 20 sept. 1729, ibid.: 384. J. Rousseau, Acoutsina, in Dictionnaire biographique du Canada (Les Presses de l'Université Laval, Québec, 1966), I: 8; M. Trudel, L'Esclavage au Canada français (Les Presses de l'Université Laval, Québec, 1960), 82.

37 Ch. de la Morandière, op. cit., I: 20.

38 J.-L. Fornel, «Relation... de la Baye des Eskimaux», op. cit.: 73. 
Malgré leur résistance parfois acharnée, les Inuit sont peu à peu refoulés hors du détroit de Belle-Isle. Jusqu'en 1720, ils reviennent bien quelquefois à la charge autour des postes des Canadiens et des emplacements de pêche des morutiers français, mais bientôt, ils n'osent plus s'aventurer vers la baye de Phélypeaux, comme ils en avaient l'habitude, préférant rester dans les hâvres de l'entrée atlantique du détroit de Belle-Isle ${ }^{39}$. Un rapport écrit vers 1760 rend compte de cette situation. On y établit «qu'il y a plus de douze ans» que les Inuit ne sont plus venus au-delà du détroit de Belle-Isle ${ }^{40}$. Les Français pensent même, à cette époque, repousser les Inuit encore plus loin le long du littoral atlantique. Fornel écrit en effet que «s'il se faisait des établissements pour la pesche sédentaire de loup-marin dans les bayes et isles qui sont le long de la coste des Eskimaux on empescherait leurs brigandages, car ces barbares ne trouvant de hâvre pour se réfugier, et pour y estre en sureté, s'éloigneraient de plus en plus, et n'oseraient approcher du détroit de BelIsle, ce qui mettrait les pescheurs, et toute la coste en sureté ${ }^{41}$.

\section{Conclusion}

La Conquête met un terme à l'exploitation canadienne et française des pêcheries au Labrador en même temps qu'aux projets de repousser encore davantage les Inuit. Toutefois, les effets de cette exploitation entre 1700 et 1760 conservent un caractère définitif: les Inuit ont été chassés de toute une partie de la côte méridionale, c'est-à-dire de la Côte-Nord du St-Laurent et du détroit de BelleIsle.

Ainsi, l'analyse des données disponibles permet de dépasser les interprétations courantes. L'hostilité entre les Amérindiens et les Inuit et la rancune de ces derniers envers les Français a pu jouer un rôle dans la nature de l'interaction entre ces groupes, notamment au $17^{\mathrm{e}}$ siècle. Durant la première moitié du $18^{\mathrm{e}}$ siècle cependant, l'expulsion des Inuit de la côte méridionale du Labrador est attribuable de façon directe et prédominante aux mesures imposées aux Inuit par suite de l'exploitation canadienne et française des pêcheries.

39 «Lettre du président du Conseil à M. de Brouage», 11 février 1737, in RAPQ (1922-23): 399.

40 Anonyme, «État des postes établis...», op. cit. : 219.

41 J.-L. Fornel, «Relation... de la Baye des Eskimaux», op. cit. : 73. 
Cette exploitation prend tôt la forme d'une occupation. Des postes sont érigés tout au long de la côte où une main-d'œuvre relativement abondante se livre à l'année longue à un ensemble d'activités littorales reliées à la pêche sédentaire au phoque. Durant la saison estivale, des sites de pêche sédentaire à la morue, surtout concentrés près du détroit de Belle-Isle, sont aussi fréquentés par un nombre considérable de pêcheurs français.

Pour les Inuit, cette période n'est pas «sans histoire» et l'histoire qu'ils tracent n'est pas celle d'une série d'événements accidentels sans lien logique les uns avec les autres. L'exploitation des pêcheries signifie l'occupation d'un territoire qu'ils fréquentent et la dépossession lente d'une de leurs principales ressources: le phoque. Leurs relations avec les pêcheurs ne leur réservent que la réduction en esclavage ou la destruction physique. Malgré une résistance acharnée et farouche, les forces en présence sont trop disproportionnées: ils doivent céder aux pressions des pêcheurs.

Deux autres conclusions - l'une méthodologique, l'autre théorique - se dégagent de cette explication. Les ethnologues qui se sont intéressés au Labrador méridional ont étudié trop exclusivement les Inuit et sous-estimé l'importance des pêcheries, tandis que les historiens eux se sont attachés à décrire ces pêcheries et ont négligé leurs effets sur les Inuit. D'où la nécessité d'abattre les cloisons entre ces disciplines jusqu'ici autonomes! Il apparait tout aussi évident que l'explication de l'expulsion des Inuit du Labrador méridional réside moins au niveau des mentalités primitives qu'au plan déterminant des rapports sociaux de production. 